\title{
Generation Scheduling of Electricity with Integrated Wind-Thermal Units using Grey Wolf Optimization Algorithm
}

\author{
R. Saravanan \\ Research scholar, \\ Department ofElectricalEngineering, \\ Annamalai University, \\ Annamalai Nagar, \\ Tamil Nadu, India \\ V. Dharamalingam \\ Professor \\ Department of \\ Electrical\&ElectronicsEngineering, \\ Pandian Saraswathi \\ Yadav Engineering College, \\ Sivagangai, \\ Tamil Nadu, India
}

\author{
S. Subramanian \\ Professor, \\ Department of ElectricalEngineering, \\ Annamalai University, \\ Annamalai Nagar, \\ Tamil Nadu, India \\ S. Ganesan \\ Assistant Professor, \\ Department of \\ ElectricalEngineering, \\ Annamalai University, \\ Annamalai Nagar, \\ Tamil Nadu, India
}

\begin{abstract}
Integrating wind power with any other energy source in power system has many operational and scheduling complications because of its inconsistent nature in the process ofwind forecasting. In this paper, a new metaheuristic optimization method named Grey Wolf Optimization algorithm is involved for solving the problem of generation scheduling (GS) to obtain best possible solution in power systems taking into account the load balance, reserve requirement, wind power availability constraints, inequality and equality constraints. The proposed GWO method is applied to a test system involves 40 conventional units and 2 wind farms. The system performance of GWO algorithm is establishedbyevaluating the results obtained for different number of trails and various iterationsfor five different populations. Calculation of the solution for different populations in the systemdiscloses that the best optimal scheduleachieved by Grey Wolf Optimization algorithm.
\end{abstract}

\section{Keywords}

Generation scheduling, Grey wolf optimization, Total generation cost reduction, Wind power availability.

\section{INTRODUCTION}

Electricity becomes the primary need for all in the world with rapid emerging technologies. Electricity demand is increasing day by day. Thus, non-conventional energy sources are needed for the generation of electricity to meet consumers overall demand effectively. Wind energy is judged to be the most reliable and promising source of electric power production in near future for the following advantages. It is clean and has no greenhouse and net carbon emissions and it is cheap and economically effective in general.

The generation scheduling problem aims to lessen the production cost of electric power under different operational constraints and physical limitations on the components of the power system.Properscheduling of unit improves the economic benefits of a power system and it is must to select the best solution method. Since large economic benefits could be achieved from unit scheduling improvement, a considerable attention has been devoted to development of related solution methods. Various mathematical programming and heuristic. Various approaches include mathematical programming and heuristic approaches such as dynamic programming [2], neural networks [3], simulated annealing [4-6], evolutionary programming [7-9] constraint logic programming [10], genetic algorithm [11-13],Lagrangian relaxation [14-16], branch and bound [17], tabu search $[18,19]$, particle swarm optimization [22-26] approaches have been devoted to solve the UCproblem. This paper considers the generation scheduling problem which includes wind power generation along with thermal generating stations. In this problem, by increasing the reserve requirements, the impacts of wind power generation are modeled when specifying the reserve inequality for this problem. The irregular nature of wind power generation in each period is replaced by wind energy speed of each period and power related to this speed. The 40 conventional units and 2 wind farms in test system is solved by the proposed GWO optimization algorithm and the results are compared among different populations and with conventional PSO method[22] to prove that GWO has better computational efficiency.

\section{PROBLEM FORMULATION}

The objective function of the GS problem is to minimize the total production cost including fuel cost, operating and maintenance cost of the generating units for the specified period under the operating constraints. The time horizon for study of this problem is one year with monthly intervals for major changes in the schedules. Due to the longer time intervals in the scheduling than the time interval of change in any generating unit, the ramp rate and minimum up/down constraints on output of the generating units are all ignored. The equation of objective function is given by, 


$$
\begin{aligned}
& \min J=\sum_{t=1}^{T} \sum_{g=1}^{N_{G}}\left\{F\left(P_{G D}(g, t)\right) \cdot n(t)\right\} \cdot U(g, t)+ \\
& \sum_{t=1}^{T} \sum_{g=1}^{N_{G}}\left\{\left(P_{G D}(g, t)+\right.\right. \\
& \left.\left.P_{G R}(g, t)\right) \cdot \text { OMVCT }(g) \cdot n(t)\right\} \cdot U(g, t)+ \\
& \sum_{t=1}^{T} \sum_{g=1}^{N_{G}}\left\{\frac{\text { PGg } \max (g) \cdot O M F C T(g) \cdot n(t)}{8760}\right\}+ \\
& \sum_{t=1}^{T} \sum_{g=1}^{N_{W}}\left\{P_{W}(w, t) \cdot \text { OMVCW }(w) \cdot n(t)\right\} \cdot V(w, t)+ \\
& \sum_{t=1}^{T} \sum_{w=1}^{N_{W}}\left\{\frac{P_{W}, \max (w) \cdot O M F C W(w) \cdot n(t)}{8760}\right\}(1)
\end{aligned}
$$

where,

$$
F\left(P_{G D}(g, t)\right)=a_{g}+b_{g} \cdot P_{G D}(g, t)+c_{g} \cdot\left(P_{G D}(g, t)\right)^{2}
$$

The equation of this objective function is subject to the number of systems and its unit constraints. The following equation should be satisfied to meet the load demand.,

$$
P d(t)=\sum_{g=1}^{N_{G}} P_{G D}(g, t) \cdot U(g, t)+\sum_{W=1}^{N_{W}} P_{W}(w, t) \cdot V(w, t)
$$

$\mathrm{t}=1,2,3 \ldots \mathrm{T}(2)$

The reserve requirement should also be satisfied. The reserve in a system is needed to provide for any feasible unpredicted generation shortage. The accuracy of the load and wind power forecasts will have a significant bearing on the system reserve levels. Increasing amounts of wind capacity causes a greater increase in the required reserve. In this paper, there are two parts in operating reserve requirement .1) Percentage of the total system load (eg., $5 \%$ of system load) 2) Surplus/Excess reserve is chosen to balance the inequality among the predicted wind electric power production and its actual value. The percentage of total wind power availability (RESW) is used in this paper to find the second part of the operating reserve. The error due to wind power forecasting is compensated using the factor (RESW). It is assumed to be $10 \%$ of the total wind power availability in each wind farm. The conventional units (40 units) in the system are responsible for both the parts of the operating reserve requirement.

$$
\begin{aligned}
\sum_{g=1}^{N_{G}} P_{G R}(g, t) \cdot U(g, t) & \geq P_{R(t)}+R E S W \\
\times & \sum_{w=1}^{N_{W}} P_{W}(w, t) \cdot V(w, t)
\end{aligned}
$$

$\mathrm{t}=1,2,3 \ldots \mathrm{T}(3)$

The generating unit constraints should also be satisfied. Therefore the equation satisfies the wind power availability is given by,

$P_{W}(w, t) \leq W_{a v}(w, t)(4)$

$\mathrm{t}=1,2,3 \ldots \mathrm{T}$

The equation showing the maximum and minimum generation in the generating units is as follows.,

$P_{G g, \min } \leq P_{G D}(g, t)+P_{G R}(g, t) \leq P_{G g, \max }(5)$

\section{WIND GENERATION MODEL}

It is necessary to accurately evaluate the electricity generated by a wind unit, located at a specific site, throughout the generation scheduling. Wind speed from $5 \mathrm{~m} / \mathrm{s}$ to about $25 \mathrm{~m} / \mathrm{s}$ is treated to be most suitable nearly for all wind turbines. With respect to the wind speed, there is some variation in the power produced.

Low Speed Region(Zero to Cut-In $\left(V_{c i}\right)$ SPEed): In this region, the turbine is kept in braked position till minimum wind speed(about $5 \mathrm{~m} / \mathrm{s}$ ), known as cut-in speed becomes available. Below this speed, the operation of the turbine is not efficient.

MAXIMUM POWER-COEFFICIENT REGION:In this region, rotor speed is varied with wind speed so as to operate it at constant tip-speed ratio, corresponding to maximum power coefficient, $C_{P M A X}$. In this range, the nature of characteristics is close to that of maximum power available in the wind and is given by,

$$
\frac{P_{o}}{A}=\frac{1}{2} \rho u_{o}^{3}(6)
$$

The turbine is operated at maximum-power-output point using pitch control.

CONSTANT POWER REgION (CONSTANT-TURBINE-SPEED REGION): During high-speed winds (above $12 \mathrm{~m} / \mathrm{s}$ ), the rotor speed is limited to an upper permissible value based on the design limits of system components. In this region, the power coefficient is lower than $C_{P M A X}$.

Furling Speed Region(Cut-Out $\left(V_{c o}\right)$ SPeEd AND AвOVE): Beyond a certain maximum value of wind speed (around $25 \mathrm{~m} / \mathrm{s}$ ), the rotor is shut down and power generation is stopped to protect the blades, generator and other components of the system. The power generated $P_{i}$ is given by,

$$
P_{i}=\left\{\begin{array}{l}
0 \\
P_{r} \\
P_{r} \\
0
\end{array} \times\left(A+B \times S W_{i}+C \times S W_{i}^{2}\right)\right\}(7)
$$

$0 \leq S W_{i}<V_{c i}$

$V_{c i} \leq S W_{i}<V_{r}$

$V_{r} \leq S W_{i} \leq V_{c o}$

$S W_{i}>V_{c o}$

Where $\mathrm{A}, \mathrm{B} \& \mathrm{C}$ are constants and are given by,

$$
\begin{array}{r}
A=\frac{1}{\left(V_{c i}-V_{r}\right)^{2}}\left\{V_{c i}\left(V_{c i}+V_{r}\right)-4 V_{c i} V_{r}\left[\frac{V c i+V r}{2 V r}\right]^{3}\right\}(8) \\
B=\frac{1}{\left(V_{c i}-V_{r}\right)^{2}}\left\{4\left(V_{c i}+V_{r}\right)\left[\frac{V c i+V r}{2 V r}\right]^{3}-\left(3 V_{c i}+V_{r}\right)\right\}(9) \\
C=\frac{1}{\left(V_{c i}-V_{r}\right)^{2}}\left\{2-4\left[\frac{V c i+V r}{2 V r}\right]\right\}(10)
\end{array}
$$

The model for wind power production is used to make a wind turbine rated $2 \mathrm{MW}$, with rated, cut-in, and cutout wind speeds of $14 \mathrm{~m} / \mathrm{s}, 2.5 \mathrm{~m} / \mathrm{s}$ and $25 \mathrm{~m} / \mathrm{s}$ respectively However for the formulation of equation for $P_{i}$ and parameters of the wind power curve, the wind speed of less than $4.3 \mathrm{~m} / \mathrm{s}$ must be expelled to avoid a wind power output of less than zero.

\section{OVERVIEW OF GWO METHOD}

The GWO is a new meta-heuristic and swarm intelligence based algorithm and it imitates the headship hierarchy and hunting method of grey wolves in nature proposed by SyedAliMirjalili, Syed Mohammad Mirjalili and Andrew Lewis. Grey Wolf Optimizer(GWO) algorithm find its application in various optimization problems such as Economic dispatch problems, Training multi-layer 
perceptron neural network, Optimal control of DC motor, Blackout risk prevention in a smart grid and Feature subset selection.

This GWO algorithm replicates the intelligent search strategy employed by the grey wolves to find the exact prey and to attack it successfully by coordinating with each other during the hunting process. Alpha, Beta, Delta and Omega are the four types of grey wolves employed for simulate the leadership hierarchy. Encircling prey, hunting, searching for prey, and attacking prey are the four important steps that are implemented to perform optimization

\subsection{Social hierarchy}

The grey wolves in general have a strict social hierarchy to mutually help out each other in hunting process and to maintain stability. The position of Alpha wolf is based on its potential and attacking capability. In this Generation Scheduling problem, the first and best fittest solution is finalized as alpha $(\alpha)$, the second and third best solutions are named beta $(\beta)$, and delta $(\delta)$ respectively. The other remaining solutions are assumed to be omega $(\omega) . \alpha, \beta$ and $\delta$ are used to guide the hunting (Optimization) in GWO algorithm. The $\omega$ solutions follow these three solutions namely $\alpha, \beta$ and $\delta$ throughout the optimization process.

\subsection{Mathematical model}

Mathematical model of hunting technique and the social hierarchy of grey wolves are needed to design and perform GWO algorithm.

\subsubsection{Encompassing prey:}

The prey is encircled by the grey wolves during the hunting process. Mathematical model of encircling action is as follows:

$$
\begin{aligned}
& \vec{E}=\left|\vec{C} \cdot \vec{X}_{P}(k)-\vec{X}(k)\right| \\
& \vec{X}_{(k+1)}=\vec{X}_{p}(k)-\vec{A} \cdot \vec{E}
\end{aligned}
$$

Where $\vec{A}$ and $\vec{C}$ are coefficient vectors and are given by:

$$
\begin{aligned}
& \vec{A}=2 \cdot \vec{a} \cdot \overrightarrow{r_{1}} \cdot \vec{a} \\
& \vec{C}=2 \cdot \overrightarrow{r_{2}}
\end{aligned}
$$

$\vec{X}$ is the position vector of grey wolves and $\vec{X}_{P}$ is the vector representing the position vector of the prey.

$r_{1}$ and $r_{2}$ are random vectors between the interval $[0,1]$ and values of $\vec{a}$ linearly varies from 2 to 0 during the iteration process.

4.2.2Hunting Mechanism: The location of prey is found by the grey wolves generally in an efficient manner and they surround it. The hunt is headed by the alpha followed by beta and delta. Remaining search agents must update their positions with respect to the position of best search agent and is mathematically formulated as,

$$
\begin{array}{r}
\quad \vec{E}_{\alpha}=\left|\overrightarrow{C_{1}} \cdot \vec{X}_{\alpha}-\vec{X}\right| \\
\vec{E}_{\beta}=\left|\overrightarrow{C_{2}} \cdot \vec{X}_{\beta}-\vec{X}\right| \\
\vec{E}_{\delta}=\left|\overrightarrow{C_{3}} \cdot \vec{X}_{\delta}-\vec{X}\right|
\end{array}
$$

$$
\begin{aligned}
& \vec{X}_{1}=\vec{X}_{\alpha}(k)-\vec{A}_{1} \cdot\left(\vec{E}_{\alpha}\right) \\
& \vec{X}_{2}=\vec{X}_{\beta}(k)-\vec{A}_{2}\left(\vec{E}_{\beta}\right) \\
& \vec{X}_{3}=\vec{X}_{\delta}(k)-\vec{A}_{3} \cdot\left(\vec{E}_{\delta}\right)
\end{aligned}
$$

Each omega wolf will update its position using the following equation.

$$
\vec{X}(k+1)=\frac{\overrightarrow{X_{1}}+\overrightarrow{X_{2}}+\overrightarrow{x_{3}}}{3}
$$

Where $\mathrm{k}$ indicates the current iteration, $X_{\alpha}(k), X_{\beta}(k)$ and $X_{\delta}(k)$ are the positions of the grey wolves $\alpha, \beta$ and $\delta$ at $k^{\text {th }}$ iteration respectively.

4.2.3Seek for prey and hitting the prey:Exploration and Exploitation are the two different abilities of the grey wolves. Seeking for the prey is the exploration ability and harassing the prey is the exploitation ability. Here an arbitrary value ' $A$ ' in between the interval [-2a,2a] is considered. If the value of $A>1$, the omega wolves should deviate its way to find the fittest prey. If the value of $A<1$, the omega wolves will attack the estimated prey by the dominant wolves.

\subsection{GWO algorithm}

Step 1: Initialize the population (n) of the grey wolves.

Step 2: Initialize the value of parameter ' $a$ ' and the value of the co-efficient vectors $\mathrm{A}$ and $\mathrm{C}$ and the current iteration value ' $k$ '.

Step 3: Initialize the maximum number of iterations along with the total number of generating units.

Step 4: Calculate the fitness of each search agent $\vec{X}_{\alpha}, \vec{X}_{\beta}$ and $\vec{X}_{\delta}$.

- $\alpha$ will be the best search agent

- $\quad \beta$ is the second best search agent

- $\delta$ is the third best search agent

Step 5: Check k<maximum no. of iteration.

Step 6: If yes, Update the position of the current search agent for each search agent $\vec{X}_{\alpha}, \vec{X}_{\beta}$ and $\vec{X}_{\delta}$ using the equation no. $8,9,10 \& 11$.

Step 7: Update the values of a, A, C and calculate the fitness of all search agents with the updated value of a, A, C. If No, Return the alpha value.

Step 8: Using the newly calculated fitness value, update the position of each search agent $\vec{X}_{\alpha}, \vec{X}_{\beta}$ and $\vec{X}_{\delta}$.

Step 9: Now again check whether $k<$ maximum number of iterations. If yes, increment the iteration number by 1 and go to step 6 .

Step 10: If k>maximum number of iterations, then stop the process and return the alpha value.

\subsection{GWO initialization procedure}

The values of $W_{\max }, W_{\min }$ and maxiter are taken as 1.0, 0.1 and 100 respectively.[values taken based on the other papers mentioned in reference [27, 28, 29]. 


\section{RESULT OF TEST SYSTEM}

The performance effectiveness of the proposed optimization algorithm (GWO) is evaluated in two parts by applied it to a model system. The two parts are; Initialization and simulation parts. Five different populations say $10,20,30,40$, and 50 in a test system are tested to find and verify the feasible optimal solution of the proposed GWO algorithm to solve the GS problem. The results obtained from GWO method in test system is compared among the results obtained for five different

populations and also with the result of conventional PSO method.
This test system has 42 generating units in total, which includes 40 conventional units and 2 wind farms (units 41 and 42$)(40 \mathrm{C}+2 \mathrm{~W})$. The input data for 40conventional units and 2 wind farms in this test system are taken from [14] [30] respectively

\subsection{Test system}

For this test system, the total load is considered as 9500MW [22]. Table 1 shows the load pattern, reserve requirement and wind farm output. Each wind farms hold 40 wind turbine units with $2 \mathrm{MW}$ capacities. Here, the value of RESW is assumed to be $10 \%$ of total wind power availability of each wind farms.

Table 1.Load pattern, reserve requirement and wind farm output

\begin{tabular}{|c|c|c|c|c|c|c|}
\hline \multirow{2}{*}{$\begin{array}{c}\text { Period } \\
\text { Month) }\end{array}$} & $\begin{array}{c}\text { Percentage of } \\
\text { annual peak } \\
\text { load (\%) }\end{array}$ & \multirow{2}{*}{$\begin{array}{c}\text { Reserve } \\
\text { Requirement } \\
(\mathbf{M W})\end{array}$} & \multicolumn{2}{|c|}{ Wind Speed (m/s) } & \multicolumn{2}{|c|}{ Wind power availability (MW) } \\
\cline { 4 - 7 } & 87.8 & 419.0683 & 5.788 & 8.284 & 3.576 & 16.607 \\
\hline 1 & 88 & 419.7905 & 5.358 & 8.149 & 2.235 & 15.675 \\
\hline 2 & 75 & 359.1935 & 5.829 & 9.446 & 3.717 & 25.718 \\
\hline 3 & 83.7 & 400.8643 & 7.193 & 9.134 & 9.817 & 23.076 \\
\hline 4 & 90 & 430.6211 & 7.989 & 8.284 & 14.60 & 16.607 \\
\hline 5 & 89.6 & 427.7703 & 7.559 & 7.19 & 11.90 & 9.798 \\
\hline 6 & 88 & 419.8043 & 7.25 & 6.826 & 10.13 & 7.913 \\
\hline 7 & 80 & 383.8324 & 7.063 & 9.836 & 9.122 & 29.202 \\
\hline 8 & 78 & 373.2626 & 7.591 & 8.127 & 12.09 & 15.529 \\
\hline 9 & 88.1 & 420.5806 & 6.165 & 8.213 & 4.937 & 16.119 \\
\hline 10 & 94 & 449.2722 & 6.414 & 8.966 & 6.007 & 21.715 \\
\hline 11 & 100 & 479.1649 & 7.035 & 10.202 & 8.973 & 32.676 \\
\hline 12 & & & & & Unit 42 \\
\hline
\end{tabular}

The optimal solution for supplying load (reserve) contribution is shown in table 2 .

Fig. 1 explains the sensitivity analysis of parameters' selection for proposed GWO in test system and Fig 2 illustrates the Convergence Characteristics of proposed GWO in test system with 100 iterations.

In this paper, the best results are verified through comparison of the results of all the five populations in the test system solved using proposed GWO method. All the results are found via a process which involves 100 trails. It is observed that the population size of 10 led to the best results, due to the best fitness and the least calculation time. The comparison of test system with the conventional PSO method [22] using the common population size 10 is tabulated in Table 4. On observing Table 4, it is clear that the total cost is reduced in test system $(40 \mathrm{C}+2 \mathrm{~W})$ solved by GWO than the total cost of GS problem solved by PSO method [22]. The research work in future will aim on the detailed development of proposed method with some improvement to deal with large scale wind power generation in the UC problem to incorporate security constraint.

Table 3. The Simulation Results For Different Population Sizes Of GWO For 100 Iterations And 100 Trails In Test System $(40 \mathrm{C}+2 \mathrm{~W})$

\begin{tabular}{|l|l|l|l|l|l|}
\hline \multicolumn{2}{|c|}{} & \multicolumn{2}{|c|}{ Total Cost (M\$) } & \multicolumn{2}{l|}{} \\
\hline $\begin{array}{l}\text { Metho } \\
\text { d }\end{array}$ & Pop & $\begin{array}{l}\text { Min. } \\
\text { Cost }\end{array}$ & $\begin{array}{l}\text { Avg. } \\
\text { Cost }\end{array}$ & $\begin{array}{l}\text { Std. } \\
\text { Dev. }\end{array}$ & $\begin{array}{l}\text { Accura } \\
\text { cy }\end{array}$ \\
\hline \multirow{2}{*}{ Size } & 10 & 881.301 & $\begin{array}{c}884.72 \\
8\end{array}$ & $\begin{array}{c}1.238 \\
7\end{array}$ & 65.234 \\
\cline { 2 - 6 } GWO & 20 & 881.312 & 885.96 & 1.299 & 63.374 \\
\hline
\end{tabular}

\begin{tabular}{|c|c|c|c|c|c|c|c|}
\hline & & & & \multicolumn{2}{|c|}{4} & 6 & \\
\hline & 30 & \multicolumn{2}{|c|}{881.321} & \multicolumn{2}{|c|}{$\begin{array}{c}886.06 \\
4 \\
\end{array}$} & $\begin{array}{c}1.275 \\
9\end{array}$ & 63.350 \\
\hline & 40 & \multicolumn{2}{|c|}{882.32} & \multicolumn{2}{|c|}{$\begin{array}{c}886.52 \\
0 \\
\end{array}$} & $\begin{array}{c}1.325 \\
7\end{array}$ & 59.113 \\
\hline & 50 & \multicolumn{2}{|c|}{883.308} & \multicolumn{2}{|c|}{$\begin{array}{c}887.73 \\
3\end{array}$} & $\begin{array}{c}1.137 \\
5\end{array}$ & 54.296 \\
\hline Pop 10 & \multicolumn{2}{|c|}{ Pop 20} & \multicolumn{2}{|c|}{ Pop 30} & \multicolumn{2}{|c|}{$\begin{array}{l}\text { Pop } \\
40\end{array}$} & \\
\hline
\end{tabular}

For illustration, reserve requirement of this test system at third period is $359.1935 \mathrm{MW}$. It is found by summing two parts. ie., 1. 356.25 (5\% of total load) and 2. 2.9427 (10\% of wind power availability). The minimum, average and standard deviation of the objective function of GS problem solved by GWO method is calculated and are tabulated in Table IIIfor five different populations. Table 3 shows the best result of this GS problem utilizing 100 iterations and 100 trails.

On comparing results of all five populations obtained by GWO method, we can conclude that the proposed GWO has a total cost which is less for population size 10 than the other populations' say $20,30,40 \& 50$. i.e., the total costvalue decreases with decrease in population size. The accuracy of the results of all trails for five different populations in GWO method is also tabulated in Table III. The equation for calculating accuracy of the results is as follows [31]:

Accuracy $=\left(\sum_{r}\left[F E(r)-F E_{\min }\right]\right) /\left(F E_{\max }-F E_{\min }\right) \forall r$ 


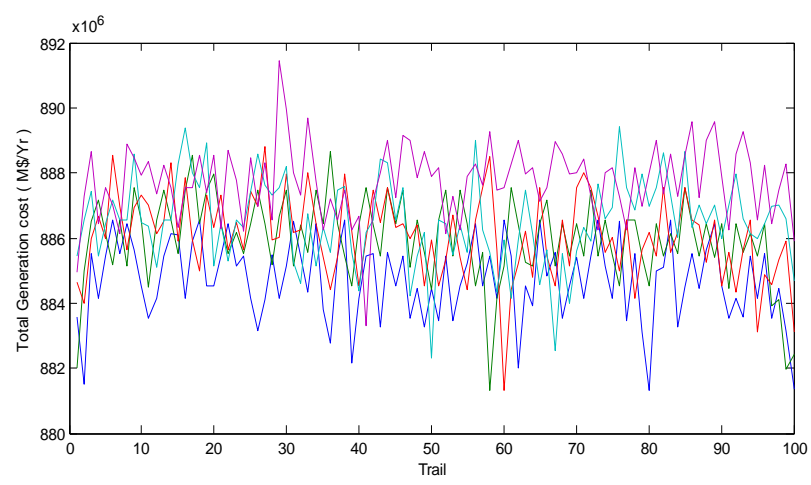

Fig 1: Sensitivity analysis of parameters' selection forproposed GWO in test system.

Table 2. Optimal Solution Result For Supplying Load (reserve) Contribution (mw) For Test System (40t+2w)

\begin{tabular}{|c|c|c|c|c|c|c|c|c|c|c|c|c|}
\hline Month & 1 & 2 & 3 & 4 & 5 & 6 & 7 & 8 & 9 & 10 & 11 & 12 \\
\hline Units & $87.8 \%$ & $88 \%$ & $75 \%$ & $83.7 \%$ & $90 \%$ & $89.6 \%$ & $88 \%$ & $80 \%$ & $78 \%$ & $88.1 \%$ & $94 \%$ & $100 \%$ \\
\hline 1 & 60.2 & 60.2 & 60.2 & 60.2 & 71.65 & 60.2 & 60.2 & 74.65 & 71.65 & 65.454 & 60.2 & 71.5 \\
\hline 2 & 78.56 & 80.4 & 65.25 & 102.5 & 82.25 & 119.36 & 101.35 & 111.54 & 112.51 & 89.45 & 109.54 & 114.65 \\
\hline 3 & 110.25 & 170.56 & 150.65 & 92.35 & 159.7 & 149.65 & 112.626 & 81.54 & 182.45 & 145.45 & 175.5 & 184.54 \\
\hline 4 & 40.23 & 41.23 & 41.26 & 25.65 & 29.65 & 32.65 & 26 & 38.45 & 32.54 & 30.241 & 36.5 & 36.5 \\
\hline 5 & 38.63 & 39.65 & 38.65 & 29.65 & 38.65 & 40.35 & 40.25 & 41.5 & 27.54 & 37.54 & 38.5 & 40.2 \\
\hline 6 & 125.65 & 127.65 & 76.25 & 134.6 & 110.7 & 120.35 & 110.54 & 125.65 & 121.45 & 111.54 & 132.54 & 125.65 \\
\hline 7 & 235.65 & 237.65 & 186.26 & 229.7 & 243.7 & 256.35 & 217.5 & 190.65 & 287.54 & 232.54 & 245.5 & 290.5 \\
\hline 8 & 198.65 & 200.03 & 216.5 & 275.7 & 197.7 & 199.35 & 148.35 & 210.65 & 296.56 & 165.54 & 280.15 & 284.54 \\
\hline 9 & 265.48 & 254.65 & 276.13 & 175.7 & 291.7 & 293.65 & 164.35 & 264.65 & 195.5 & 298.54 & 245.4 & 224.5 \\
\hline 10 & 286 & 276.26 & 265.65 & 245.7 & 292.7 & 290.33 & 202.35 & 195.54 & 185.15 & 245.45 & 212.15 & 295.54 \\
\hline 11 & 300.26 & 298.65 & 220.26 & 300.7 & 365.7 & 355.5 & 186.35 & 220 & 104.54 & 212.45 & 325.5 & 345.54 \\
\hline 12 & 302.36 & 304.26 & 295 & 119.7 & 302.7 & 298.55 & 350.245 & 274.54 & 300.54 & 110.241 & 365.54 & 326.45 \\
\hline 13 & 405.02 & 403.26 & 165.22 & 389.7 & 415.7 & 400.5 & 199.35 & 348.65 & 212.02 & 284.42 & 398.46 & 498.15 \\
\hline 14 & 323.27 & 321.568 & 405.66 & 456.7 & 396.7 & 392.22 & 412.65 & 135.15 & 178.65 & 226.54 & 454.54 & 478.1 \\
\hline 15 & 365.65 & 363.265 & 325.54 & 304.7 & 295.7 & 293.35 & 349.65 & 175.15 & 398.35 & 415.45 & 395.65 & 448.5 \\
\hline 16 & 496.35 & 391.245 & 169.03 & 208.7 & 342.7 & 340.33 & 398 & 195.2 & 321.35 & 312.45 & 313.65 & 365.5 \\
\hline 17 & 228.65 & 384.685 & 376.65 & 195.6 & 299.7 & 301.35 & 395.65 & 265.45 & 298.5 & 485.54 & 265.45 & 314.54 \\
\hline 18 & 440.27 & 338.54 & 332.26 & 320.7 & 322.9 & 327.35 & 364.5 & 410.54 & 346.15 & 412.5 & 445.65 & 302.54 \\
\hline 19 & 465.36 & 462.658 & 356.26 & 496.7 & 392.4 & 396.5 & 486.65 & 498.65 & 263.65 & 365.45 & 378.65 & 298.54 \\
\hline 20 & 533.65 & 330.25 & 283.54 & 502.6 & 365.7 & 367.35 & 321.54 & 367.54 & 305.65 & 402.15 & 483.65 & 398.65 \\
\hline 21 & 383.99 & 385.658 & 249.85 & 296.7 & 339.7 & 335.54 & 435.65 & 300.6 & 256.65 & 385.65 & 356.65 & 356.54 \\
\hline 22 & 371 & 302.257 & 294.54 & 523.4 & 335.7 & 421.35 & 524.25 & 412.5 & 514.65 & 374.305 & 365.65 & 495.65 \\
\hline
\end{tabular}




\begin{tabular}{|c|c|c|c|c|c|c|c|c|c|c|c|c|}
\hline 23 & 267.98 & 366.652 & 359.65 & 411.7 & 386.7 & 377.35 & 428.025 & 378.56 & 321.54 & 298.54 & 548.5 & 465.54 \\
\hline 24 & 279.45 & 481.654 & 306.8 & 318.7 & 362.7 & 373.03 & 513.65 & 408.65 & 294.35 & 532.5 & 369.65 & 412.65 \\
\hline 25 & 365.01 & 323.265 & 397.6 & 396.7 & 345.7 & 343.25 & 299.35 & 384.54 & 309.65 & 478.5 & 314.54 & 515.52 \\
\hline 26 & 313.05 & 347.054 & 395.65 & 315.7 & 495.7 & 392.23 & 396.65 & 365.65 & 318.54 & 398.54 & 395.65 & 512.65 \\
\hline 27 & 402.35 & 399.65 & 278.54 & 351.5 & 412.7 & 509.65 & 319.65 & 410.5 & 477 & 410.727 & 320.65 & 465.4 \\
\hline 28 & 80.06 & 75.154 & 30.2 & 119.7 & 90.66 & 87.35 & 106.4 & 104.36 & 20.65 & 95.65 & 78.65 & 106.65 \\
\hline 29 & 70.06 & 73.154 & 20.5 & 90.46 & 134.7 & 130.35 & 71.245 & 26.35 & 70.54 & 145.215 & 95.65 & 118.65 \\
\hline 30 & 64.035 & 68.265 & 12.35 & 32.65 & 115.4 & 111.25 & 118.254 & 141.35 & 80.65 & 110.15 & 141.51 & 95.007 \\
\hline 31 & 50.134 & 60.254 & 56.35 & 50.65 & 68.65 & 65.224 & 29.35 & 58.35 & 56.65 & 65.45 & 65.65 & 60.54 \\
\hline 32 & 23.658 & 25.35 & 43.26 & 60.96 & 40.66 & 37.54 & 66.25 & 60.9 & 68.35 & 36.5 & 59.65 & 68.54 \\
\hline 33 & 56.32 & 66.325 & 32.658 & 22.35 & 68.12 & 55.35 & 43.35 & 22.025 & 30.835 & 55.15 & 48.65 & 30.265 \\
\hline 34 & 60.493 & 61.25 & 40.65 & 36.65 & 32.65 & 28.54 & 36.65 & 20.862 & 69.65 & 68.54 & 36.98 & 45.54 \\
\hline 35 & 19.5 & 19 & 43.528 & 45.27 & 45.98 & 40.254 & 50.65 & 42.025 & 20.31 & 36.45 & 54.65 & 19.45 \\
\hline 36 & 22.05 & 19.658 & 21.356 & 20.01 & 35.65 & 30.5 & 19.025 & 20.013 & 57.65 & 31.65 & 44.65 & 26.54 \\
\hline 37 & 32.265 & 40.65 & 23.6 & 28.33 & 27 & 23.056 & 21.025 & 25.024 & 45.987 & 45.94 & 57.65 & 30.25 \\
\hline 38 & 26.54 & 27.439 & 28.259 & 49.06 & 31 & 26.896 & 33.244 & 52.024 & 31.045 & 38.65 & 53.987 & 27.5 \\
\hline 39 & 31.015 & 28.65 & 29.65 & 28.01 & 56.27 & 33.023 & 28.006 & 27.025 & 32.895 & 28.654 & 41.028 & 58.65 \\
\hline 40 & 41.111 & 30.25 & 30.003 & 31.03 & 27.27 & 35.025 & 46.025 & 26.003 & 27.045 & 29.784 & 35.985 & 51.65 \\
\hline 41 & 20.3 & 53.25 & 60.35 & 19.65 & 58.37 & 8.356 & 75.65 & 36.65 & 18.054 & 21.654 & 59.65 & 35.54 \\
\hline 42 & 60.5 & 18.5 & 63.45 & 35.65 & 21.65 & 11.658 & 49.55 & 50.35 & 45.02 & 32.365 & 21.65 & 56.658 \\
\hline $\begin{array}{c}\text { Total } \\
\text { Cost } \\
(\mathrm{M} \$ / \mathrm{Yr})\end{array}$ & 886.32 & 886.95 & 881.26 & 884.96 & 889.55 & 887.44 & 887.65 & 883.56 & 882.46 & 887.98 & 892.68 & 894.78 \\
\hline
\end{tabular}

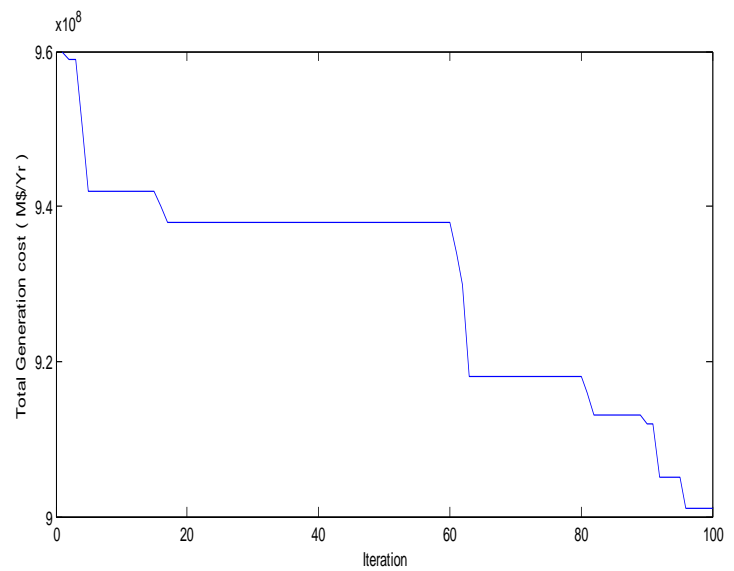

Fig 2: Convergence Characteristics of proposed GWO in test system with 100 iterations.
Table 4.Best results in different test systems of GWO along with PSO for 100 trails in different test cases

\begin{tabular}{|c|c|c|c|c|c|c|}
\hline \multirow{2}{*}{$\begin{array}{l}\text { Test } \\
\text { System }\end{array}$} & \multirow{2}{*}{$\begin{array}{l}\text { Meth } \\
\text { od }\end{array}$} & \multirow{2}{*}{$\begin{array}{l}\text { Pop. } \\
\text { Size }\end{array}$} & \multicolumn{3}{|c|}{ Total Cost(M\$) } & \multirow{2}{*}{$\begin{array}{l}\text { Acc } \\
\text { ura } \\
\text { cy } \\
(\%)\end{array}$} \\
\hline & & & $\begin{array}{l}\text { Min. } \\
\text { cost }\end{array}$ & $\begin{array}{l}\text { Avg. } \\
\text { Cost }\end{array}$ & $\begin{array}{l}\text { Std. } \\
\text { Dev. }\end{array}$ & \\
\hline $\begin{array}{c}40 \mathrm{C}+2 \\
\mathrm{~W}\end{array}$ & PSO & 10 & $\begin{array}{c}883.828 \\
8 \\
\end{array}$ & $\begin{array}{c}910.64 \\
16 \\
\end{array}$ & $\begin{array}{c}8.51 \\
23 \\
\end{array}$ & $\begin{array}{l}58.6 \\
39 \\
\end{array}$ \\
\hline $\begin{array}{c}40 \mathrm{C}+2 \\
\mathrm{~W}\end{array}$ & GWO & 10 & 881.301 & $\begin{array}{c}884.72 \\
8 \\
\end{array}$ & $\begin{array}{c}1.23 \\
87 \\
\end{array}$ & $\begin{array}{l}65.2 \\
34 \\
\end{array}$ \\
\hline
\end{tabular}

\section{CONCLUSION}

This paper presents a new optimization method for solving the generation scheduling (GS) problem based on the GWO algorithm. A new position update tactic that is integrated in the GWO method is employed to satisfy the constraints by the solutions of this problem. The output of GWO method in test system( $40 \mathrm{C}+2 \mathrm{~W}$ )is compared with the results of five different pollutions say $10,20,30,40 \&$ 50. The above simulation results show that the proposed meta-heuristic and swarm intelligence based GWO 
algorithm has better computational efficiency and it is shown that the Grey wolf Optimizer (GWO) algorithm obtains near optimal solution for GS problems. Future research work will focus on some other approach with better improvement to incorporate security constraints.

\section{REFERENCES}

[1] Ouyang, Z, Shahidehpour S.M.1991.An intelligent dynamic programming for unit commitment application,IEEE Transaction on power systems. 6(3) 1203.

[2] Sasaki, H, et.a..1002E. 2005. A solution method of unit commitment by artificial neuraltabu-search-based hybrid-optimisation technique', IEEProc.,Gener. Transm. Distrib., 152, (4), . 563-574

[3] Annakkage,U.D,et al.. 1995. Unit commitment by parallel simulated annealing, proc. Inst. Elect. Eng., Gen.Transm.Dist .142-595.

[4] Purushothama, G.K., and Jenkins, L. 2003: 'Simulated annealing with local search. A hybrid algorithm for unit commitment', IEEE Trans., PWRS$18,(1), 273-278$.

[5] Simopoulos, D.N., Kavatza, S.D., and Vournas, C.D.2006: 'Unit commitment by an enhanced simulated annealing algorithm', IEEETrans. , PWRS$21,(1), .68-76$.

[6] Lau, T.W., Chung, C.Y., Wong, K.P. 2009, et al.: Quantum inspired evolutionary algorithm approach for unit commitment',IEEETrans. Power Syst.,24(3) $.1503-1512$

[7] Patra,S.Goswami,S.K., Goswami, B.2008: Differential evolution algorithm for solving unit commitment with ramp constraints, Elect. Power compon.syst.,36.(8), 771-787.

[8] Dilip, D., Sapatarshi, D.2012:`A binary -real -coded differential evolution for unit commitment problem', Elect Power Syst.,,42,517-524.

[9] Huang.K.Y, yang.H.T, Yang.C.L. 1988, A new thermal unit commitment approach using constraint logic programming, IEEE Trans. Power syst. 13 (3).

[10] S.A. Kazarlis, A.G. Bakirtzis, V.Petridid,(1996) A genetic algorithm solution to the unit commitment problem,IEEE Trans .Power syst.11(1)83.

[11] WaltersD.C and Sheble.G.B .1993, "Genetic algorithm solution of economic dispatch with the valve-point loading", IEEE Trans.on Power Systems, Vol. 8, No. 3, 1325-1332

[12] Bakirtzis, A.G., and Petridis, V.1996 'A genetic algorithm solution to the unit commitment problem', IEEE Trans., , PWRS-11, (1), 83-92

[13] Virmani. S, et.al. 1989, Implementation of a Lagrangian relaxation based unit commitment problem, IEEE Trans. Power syst. 4(4). 1373

[14] Cheng.C. P,.Liu C. W, Liu.C.C. 2000, Unit commitment by lagrangian relaxation and genetic algorithms, IEEE Trans. Power Syst.15(2)707.

[15] Thillainathan,Logenthiran,WaiLokWoo, Van Tung Phan,Lagrangian relaxation hybrid with evolutionary algorithm with short term scheduling, Electrical power and Energy Systems., 64356-364.

[16] Cohen. A . I, Yoshimura. M, A 1983 branch - and a bound algorithm for unit commitment, IEEE Trans.PowerApp .Syst., PAS-102(2) 444

[17] Mori. H, Matsuzaki. O. 2001, Application of priority -list-embedded tabu search to unit commitment in power systems,Inst.Elect.Eng.Jpn.121-B(4)535.

[18] Juste, K.A., Kiat, H., Tanaka, E., and Hasegawa. 2005, J.: 'Unit commitment by a tabu-search-based hybrid-optimisation technique', IEEProc.,Gener. Transm. Distrib., 152, (4), pp. 563-574

[19] Karki. R. 2007, "Renewable Energy Credit Driven Wind Power Growth for System Reliability”, Electric Power System Research, vol. 77, 797-803.

[20] Hetzer J, Yu DC. 2008 An economic dispatch model incorporating wind power. IEEE Transactions on Energy Conversion; 23:603-611.

[21] ChenC.-L., Mar. 2008, "Optimal wind-thermal generating unit commitment, "IEEE Trans. Energy Convers., vol. 23, no. 1, pp. 273-280.

[22] Siahkali.H,Vakilian.M(2009),"Electricity generation scheduling with large-scale wind farms using particle swarm optimization", Electric Power Systems Research 79 826-836.

[23] Jeong, Y-W., Park, J-B., Jang,S-H.2010, et al.: `A new quantum inspired binary PSO application to unit commitment problems for power systems', IEEE Trans Power syst., ,25,(3),pp.1486-1495.

[24] Xiang Yu,Xueqing,(2014)'Unit commitment using lagrangian and particle swarm optimization', Electrical power and Energy Systems., 61510-522.

[25] AnupShukla,.SinghS.N(2016), Advanced three stage pseudo-inspired weight improved crazy particle swarm optimization for unit commitment', Electrical power and Energy Systems., 9623-36.

[26] Marian Marcoveccio, G., Augusto Novals, Q2014., Ignacio Grossmann, E `Deterministic optimization of the thermal unit commitment problem: a branch and cut search'. ComputChem, Eng.,,67,pp53-68.

[27] . GaingZ.L,2003 Discrete particle swarm optimization algorithm for unit commitment, in: IEEE PES General Meeting, , p.418

[28] GaingZ.L.,(2003) Particle swarm optimization to solving the economic dispatch considering the generator constraints, IEEE Trans. Power Syst. 18 (3) 1187-1195.

[29] Eberhart.R, Shi.R,(2000), Comparing inertia weights and constriction factors in particle swarm optimization, in:proc. Congr. Evolu. Comp. p. 84.

[30] ChenP.H, ChangH.C.(1995), Large-scale economic dispatch by genetic algorithm, IEEE Trans. Power Syst. 10 (4) 1919-1926.

[31] EngelEngelbrech.A.P,2005Fundamentalsof Computational swarm intelligence, John wiley\& Sons, England 


\section{NOMECLATURE}

$\mathrm{FE}(\mathrm{r}) \quad$-Objective function valuation at trail $\mathrm{r}$

$F E_{\max } \quad$-Maximum cost among $\mathrm{r}$ trail values

$F E_{\text {min }} \quad$-Minimum cost among $r$ trail values

g $\quad$-Index for thermal generator unit

Maxiter -Maximum number of iterations

$\mathrm{n}(\mathrm{t}) \quad$-Number of hours in time t.

$N_{G} \quad$-Number of thermal generator units

$P_{R}(t) \quad$-A fraction of total system load for system

reserve requirement (first part) at time $\mathrm{t}(\mathrm{MW})$.

RESW -A fraction of total wind power employed to compensate wind power prediction errors (\%).

$P_{w}(w, t)$-Generation of wind unit w at time $\mathrm{t}(\mathrm{MW})$. t-Index for time.

$P_{G g, \max } \quad$-Upper limit of thermal unit g (MW)

$P_{G D}(g, t)$-Load contribution of thermal unit $\mathrm{g}$ at time $\mathrm{t}$ (MW)
$P_{G g, \min } \quad$-Lower limit of thermal unit g (MW)

OMFCW (w)-Operation and maintenance fixed cost of wind unit w (\$/MW yr)

OMVCW (w)-Operation and maintenance variable cost of wind unit $\mathrm{w}(\$ / \mathrm{MWh})$.

OMVCT (g)-Operation and maintenance variable cost of thermal unit $\mathrm{g}(\$ / \mathrm{MWh})$

$W_{a v}(w, t)$-Maximum available wind power of wind unit $\mathrm{w}$ at time $\mathrm{t}(\mathrm{MW})$.

w- index for wind unit.

OMFCT (g)-Operation and maintenance fixed cost of thermal unit $\mathrm{g}(\$ / \mathrm{MW}$ yr)

$\mathrm{V}(\mathrm{w}, \mathrm{t})$-Commitment state of wind unit $\mathrm{w}$ at time $\mathrm{t}(\mathrm{on}=1$, off $=0$ ).

$N_{w}$-Number of wind units

$\mathrm{U}(\mathrm{g}, \mathrm{t})$-Commitment state of unit $\mathrm{g}$ at time $\mathrm{t}(\mathrm{on}=1$, off $=0$ )

T-Number of periods under study 